\title{
Original article \\ Lidocaine patch for acute pain management: a meta-analysis of prospective controlled trials
}

Yaowu Bai

Department of Anesthesiology, Tangshan Maternal and Child Healthcare Hospital, Hebei United University, Tangshan, China

\section{Timothy Miller}

Department of Anesthesiology, Duke University Medical Center, Durham, NC, USA

Mingjuan Tan

Duke-NUS Graduate Medical School, Singapore

\section{Lawrence Siu-Chun Law}

Tong Joo Gan

Department of Anesthesiology, Duke University Medical Center, Durham, NC, USA

Address for correspondence:

Tong Joo Gan MD, Department of Anesthesiology, Stony Brook Medicine, Stony Brook,

NY 11794-8480, USA.

tong.gan@stonybrookmedicine.edu

\section{Keywords:}

Acute pain - Lidocaine patch - Opioid sparing Postoperative pain

Accepted: 30 September 2014; published online: 28 January 2015 Citation: Curr Med Res Opin 2015; 1-7

\section{Abstract}

\section{Background:}

Local anesthetic is one of the cornerstones of multimodal analgesia. We investigated the efficacy of the lidocaine patch for acute pain management.

\section{Methods:}

We searched MEDLINE, CINAHL, Scopus, and the Cochrane Controlled Trials Register for published prospective controlled clinical trials that evaluated the analgesic effect of the lidocaine patch for acute or postoperative pain management (1966-2014). The outcomes were postoperative opioid consumption, pain intensity and length of hospital stay.

\section{Results:}

Five trials comparing the lidocaine patch with control (no treatment/placebo) for acute or postoperative pain treatment/management were included in this meta-analysis. Data was analyzed on 251 patients. Between the lidocaine patch group and the control group, no significant difference was found for all three outcomes (all $p>0.05$ ). For postoperative opioid consumption, mean difference (MD) was $-8.2 \mathrm{mg}$ morphine equivalent $(95 \% \mathrm{Cl}-28.68,12.24)$. For postoperative pain intensity, $\mathrm{MD}$ was $-9.1 \mathrm{~mm}$ visual analog scale or equivalent $(95 \% \mathrm{Cl}-23.31,5.20)$. For length of hospital stay, $\mathrm{MD}$ was -0.2 days $(95 \% \mathrm{Cl}-0.80$, 0.43).

\section{Conclusion:}

Application of a lidocaine patch may not be an effective adjunct for acute and postoperative pain management, in terms of pain intensity, opioid consumption and length of hospital stay.

\section{Limitations:}

The limitations were a small number of included studies, potential biases from some unblinded studies, clinical heterogeneity between studies, and incomplete reported data for adjunct analgesics.

\section{Introduction}

Acute pain is defined as 'pain of recent onset and probable limited duration'. It has an identifiable causal and temporal relationship to injury, surgery, or disease. Acute pain can lead to patient anxiety, stress and dissatisfaction ${ }^{1-3}$. Inadequately treated pain may cause organ dysfunction, and can lead to detrimental psychological, economic and social effects ${ }^{2,4}$. Adequate pain control should be achieved not only for humanitarian reasons, but is also because it is an important goal to allow patients to resume normal activities ${ }^{1}$.

Evidence suggests that multimodal analgesia, where combinations of two or more analgesics with different mechanisms of action are administered, is more effective than monotherapy. Multimodal analgesia techniques provide 
improved analgesia with lower doses than would be possible with a single analgesic, reduce opioid related side effects, and shorten length of hospital stay ${ }^{5,6}$.

Local anesthetics have been widely used as part of a multimodal technique. There are many routes of administration for lidocaine, such as topical, intravenous, localized subcutaneous or submucosal infiltration, spray, nerve blocks, transdermal patches, etc. Of those routes, intravenous lidocaine has been extensively researched. Two metaanalyses ${ }^{7,8}$ show that intravenous lidocaine significantly reduces postoperatively pain, opioid consumption, length of stay, and gastrointestinal recovery time. The choice of the route of administration is dependent upon several factors, including the site of surgery or injury, intended duration of action, ease of use, expertise required as well as potential adverse effects.

The topical lidocaine $5 \%$ patch is approved by the US Food and Drug Administration for the treatment of persistent neuropathic pain syndrome and postherpetic neuralgia. However, its use has been reported in a number of other painful conditions ${ }^{9-11}$. The benefits of using a lidocaine patch for the management of acute pain remain unclear. Only a few studies have investigated the efficacy of lidocaine patches on acute pain, and no meta-analysis has been conducted ${ }^{12,13}$. We therefore conducted this meta-analysis to evaluate the efficacy of lidocaine patches for acute or postoperative pain management. Our hypotheses were that the use of a lidocaine patch reduced pain scores and opioid consumption (primary outcomes) as well as length of hospital stay (secondary outcome).

\section{Materials and methods}

A systemic search was performed without language limitations. We searched MEDLINE, PUBMED, CINAHL, Scopus, and the Cochrane Controlled Trials Register for published prospective controlled clinical trials that evaluated the analgesic effect of lidocaine patches for acute or postoperative pain management (1966 - July 2014). We used free text and the MeSH terms lidocaine, Lidoderm, lignocaine, lidocaine patch, postoperative, analgesia, and acute pain. We recruited additional trials from bibliographies of the retrieved trials and previous reviews. We excluded data from abstracts, case reports, letters, and reviews. Only controlled trials of the lidocaine patch for postoperative analgesia and acute pain management in adults, with a no-treatment or a placebo group, were considered. From these, we extracted information on pain intensity, opioid consumption, and length of hospital stay.

Two reviewers independently read the titles and abstracts of the manuscript to determine whether it met the inclusion criteria $(\mathrm{kappa}=1.0)$. We sought to contact the authors on data that were not reported in the manuscript.
The primary outcomes of this systematic review were pain scores at 24 hours after the first application of the lidocaine patch and cumulative opioid consumption converted to intravenous morphine equivalent doses ${ }^{14}$ over 24 hours. The secondary outcome was the length of stay in hospital. The visual analog scale (VAS, 0-100 mm) was used as a measure of pain intensity $(0=$ no pain and $100=$ worst pain possible), and scores were analyzed quantitatively. Pain intensity score on the numeric rating scale (NRS, 0-10) was converted to a $0-100 \mathrm{~mm}$ VAS score for analysis $(V A S=N R S \times 10 \mathrm{~mm})$. Opioid analgesics were converted to intravenous morphine equivalent doses (in $\mathrm{mg})^{14}$. If trials did not report data at our prespecified study time points, we selected the closest time point for analysis. We contacted authors by e-mail or phone if additional information was needed.

Our meta-analysis was performed with Review Manager (version 5.2, Cochrane collaboration). For continuous data, mean differences (MD) were analyzed by the inverse variance method. If the $95 \% \mathrm{CI}$ included zero, the difference between the lidocaine patch and control groups was not considered as statistically significant. When mean with $\mathrm{SD}$ values were not given, they were estimated from the median and $\mathrm{SE}$ or $\mathrm{CI} \quad(\mathrm{SEM}=\mathrm{SD} / \mathrm{V} N$ and $\mathrm{SEM}=(95 \% \mathrm{CI}) / 1.96)$, or from the inter-quartile range if the data distribution was not skewed. Heterogeneity between datasets were assessed by $I^{2}$ and tested by a chisquare $\left(\chi^{2}\right)$ test. Random effects models were used if $I^{2}>25$ or $p<0.05$ for the $\chi^{2}$ test. A $p$-value $<0.05$ was considered statistically significant.

\section{Results}

A total of 87 trials were identified from the initial search, of which 82 were excluded, as they were not central to the topic of interest. Five controlled trials comparing a lidocaine patch with placebo or no treatment for postoperative analgesia or acute pain treatment/management were included in this meta-analysis. Data were available on 251 patients. There were four randomized controlled trials ${ }^{15-18}$ and one prospective cohort study ${ }^{19}$. For the prospective cohort study, during the first 6 month period patients received the lidocaine patch, with patients from the subsequent 6 month period serving as controls. The characteristics of the included studies ${ }^{15-19}$ are shown in Table 1. The flow diagram of the search process is shown in Figure 1. Table 2 presents the risk of biases and Jadad scores of included studies.

\section{Opioid consumption}

Three of the five studies provided information on opioid consumption ${ }^{15-18}$. Two studies did not provide specific information on opioid consumption ${ }^{18,19}$. Combined data 


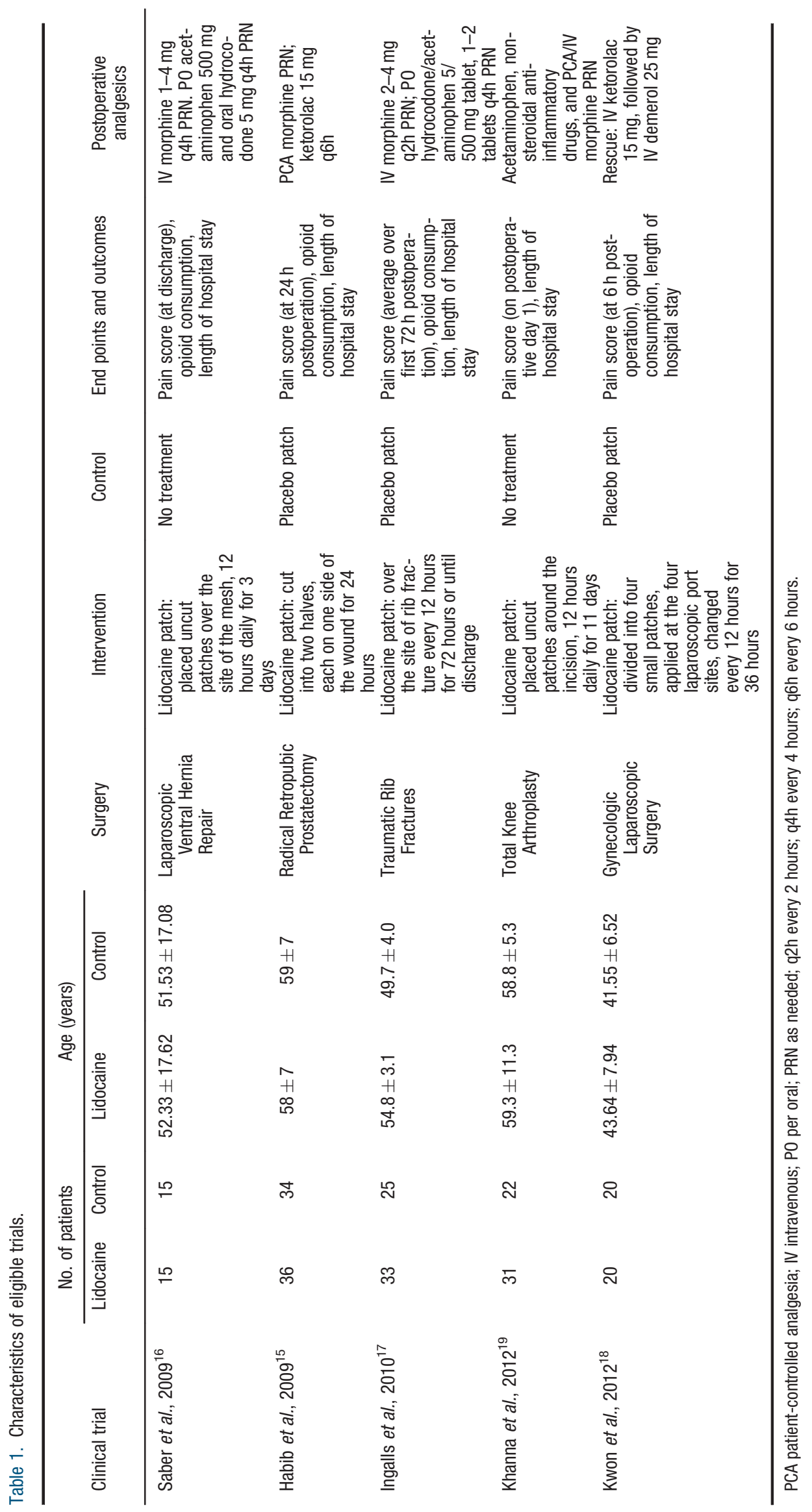


Databases: PudMed, Medline, CENTRAL, EMBASE, and CINAHL

Search: lidocaine, Lidoderm, lignocaine, lidocaine patch, postoperative, analgesia, and acute pain

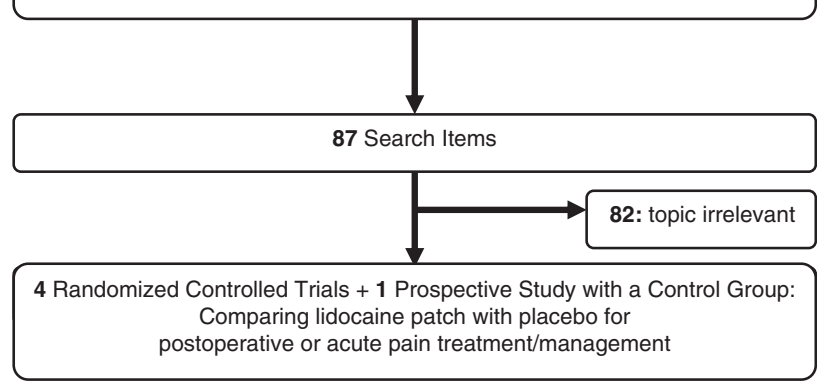

Figure 1. Flow diagram of included studies.

from three studies showed no significant difference in opioid consumption between the lidocaine patch group and the control group (MD: $-8.2 \mathrm{mg}[95 \% \mathrm{CI}$ : -28.68 , 12.24], $Z=0.79, p=0.43 ; I^{2}=88 \%$ ) (Figure 2).

\section{Postoperative pain}

All five trials provided information on pain scores at 24 hours ${ }^{15-19}$. Combined data showed no significant difference in pain intensity at rest between the lidocaine patch group and the control group $(\mathrm{MD}=-9.1 \mathrm{~mm}$ [95\% CI: $\left.-23.31,-5.20], Z=1.24, p=0.21 ; I^{2}=99 \%\right)$ (Figure 3).

\section{Length of hospital stay}

All five studies reported data for the length of hospital stay (no ambulatory/day surgery). Combined data showed no significant difference in hospital stay between the lidocaine patch group and the control group $(\mathrm{MD}=-0.2$ days [ $[95 \% \mathrm{CI}:-0.80,0.43], Z=0.60, p=0.55 ; I^{2}=43 \%$ ) (Figure 4).

\section{Adverse events}

In all five trials, use of the lidocaine patch was not associated with any adverse effects, such as wound complications, contact dermatitis and systemic side effects.

\section{Sensitivity analysis}

We repeated all analysis without the prospective cohort study ${ }^{19}$ and the results were similar.

\section{Publication biases}

Funnel plots of all three outcome variables are shown in Figures 2-4.

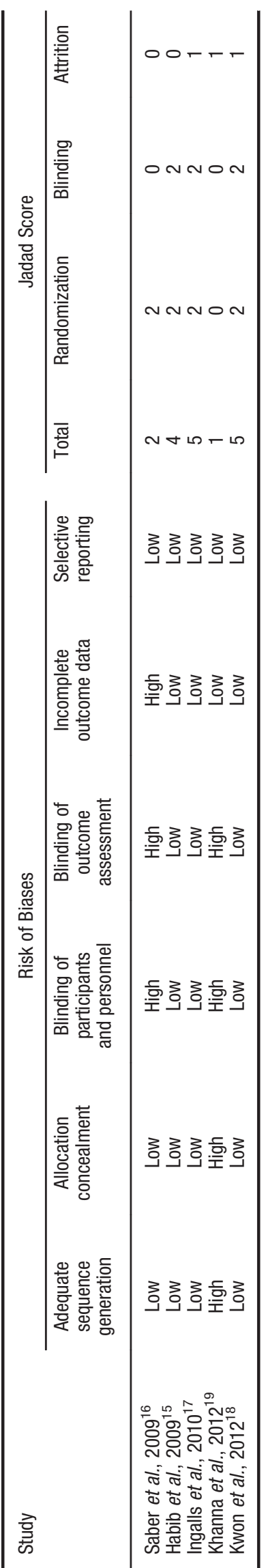




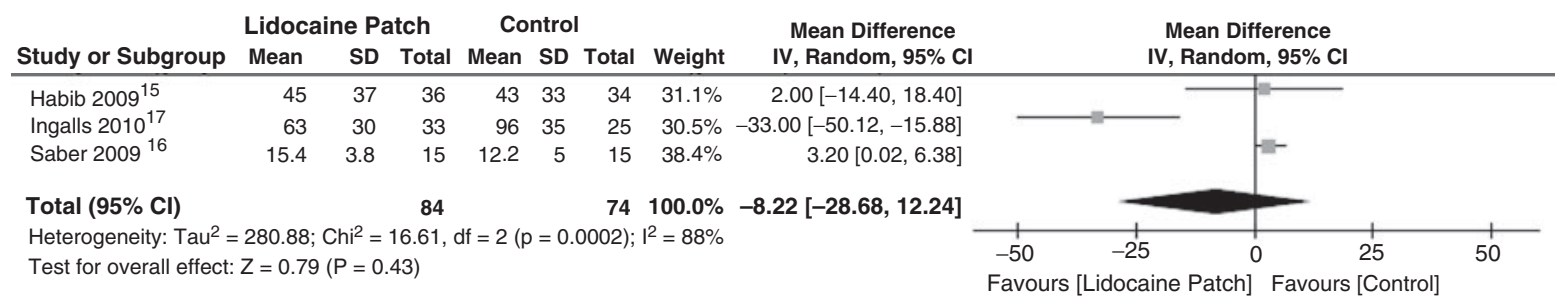

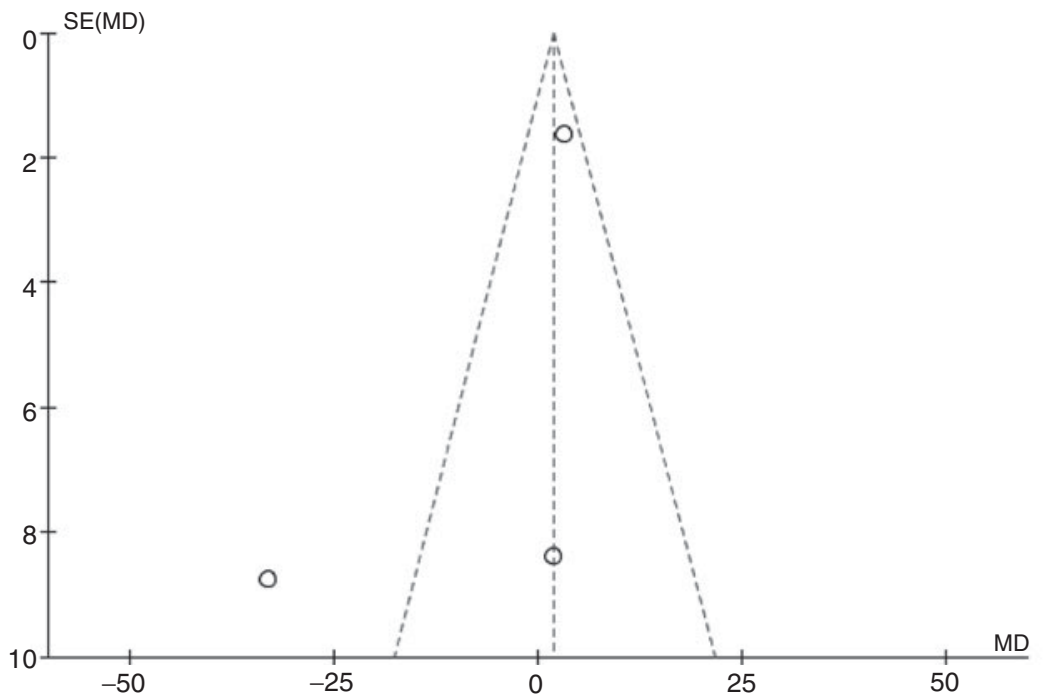

Figure 2. Cumulative opioid consumption (morphine equivalent, mg).

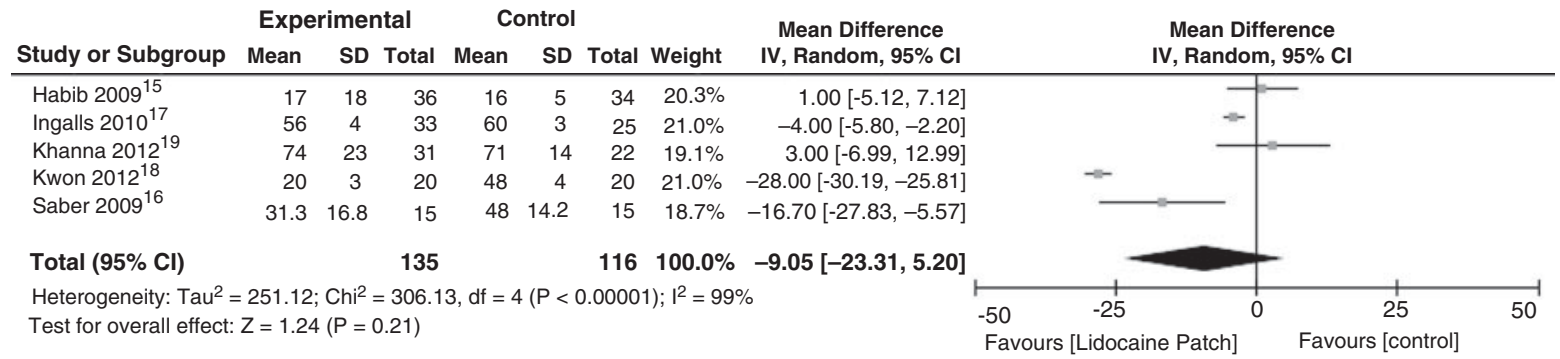

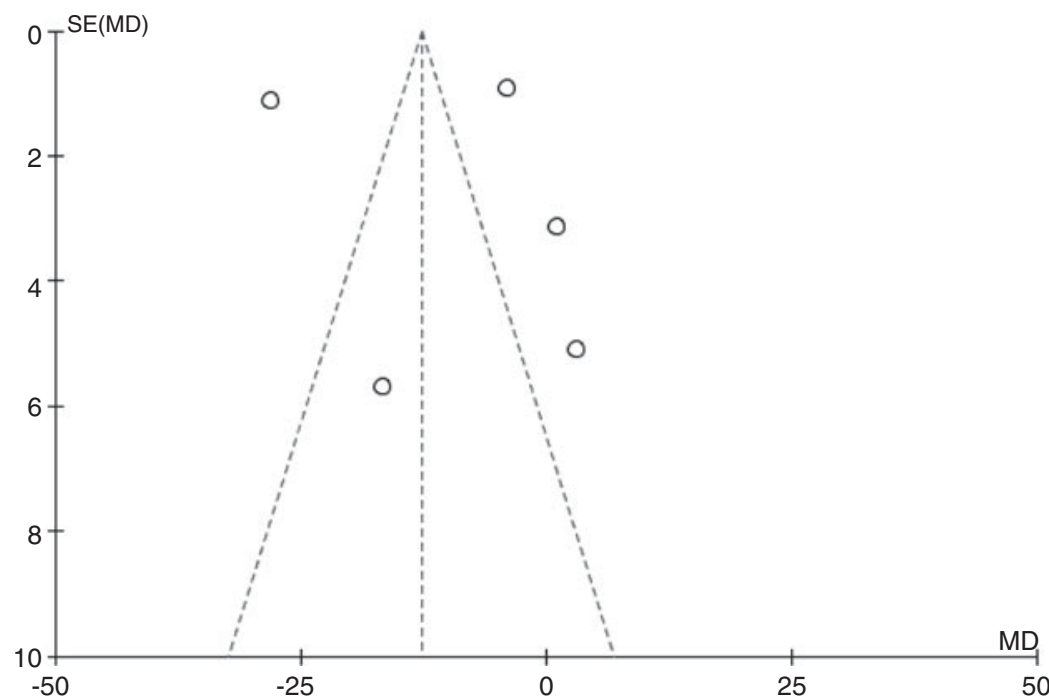

Figure 3. Pain scores (visual analog scale or equivalent, $\mathrm{mm}$ ). 


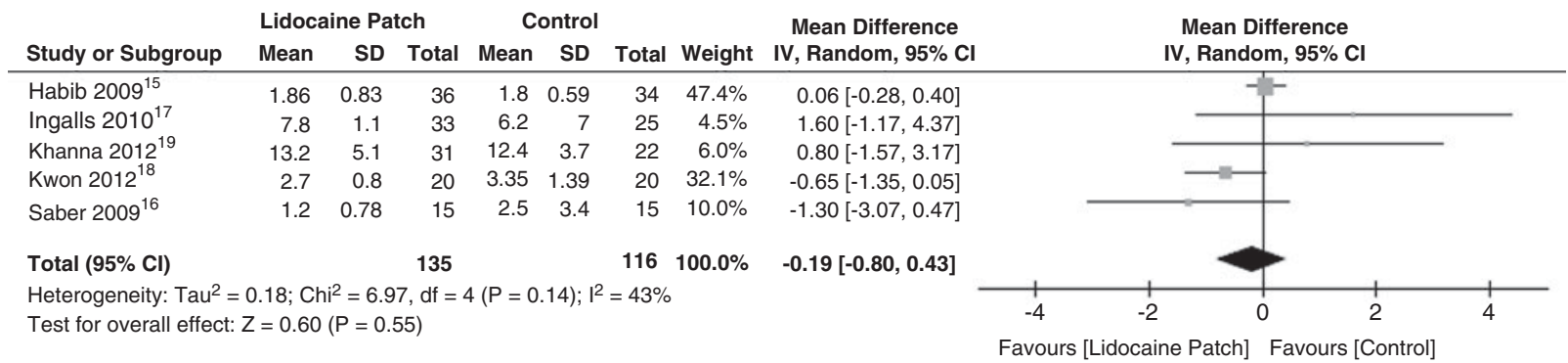

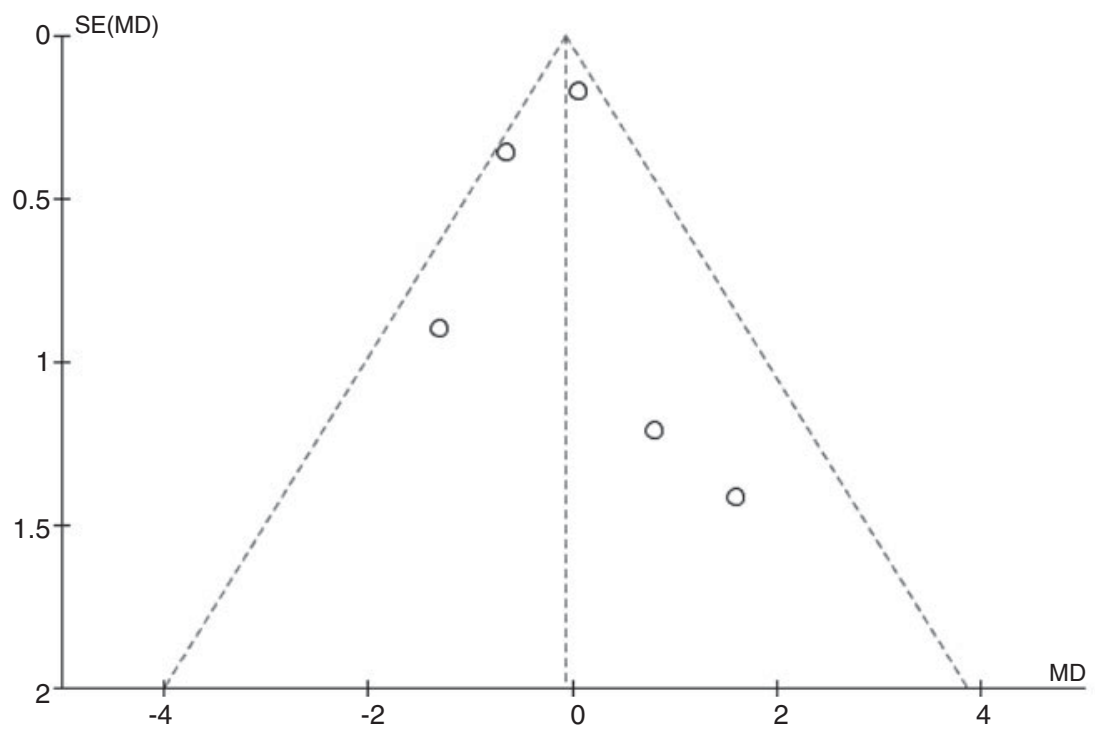

Figure 4. Length of hospital stay (days).

\section{Discussion}

This meta-analysis showed that application of a lidocaine patch did not significantly reduce postoperative pain scores, opioid consumption, or length of hospital stay. Although the patch is associated with minimal systemic absorption and side effects, it might not be an effective adjunct for the management of acute pain.

We postulated three major reasons for the insignificant results. First, the lidocaine patch delivers lidocaine to the intact skin around the wound instead of directly into the wound (in contrast with wound infiltration). Second, the lidocaine diffuses from the epidermis to the deeper parts of skin instead of spreading under the epidermis or even under the dermis like wound infiltration. Third, the lidocaine patch has minimal systematic absorption. It has fewer systemic side-effects but also less analgesic effect than intravenous lidocaine. Therefore, the lidocaine patch might not reliably reduce postoperative pain and thus did not affect the opioid consumption and length of hospital stay.

The topical lidocaine $5 \%$ patch is a $10 \mathrm{~cm}$ by $14 \mathrm{~cm}$ soft, stretchy adhesive patch that contains $700 \mathrm{mg}$ of $5 \%$ lidocaine in an aqueous base. It delivers the drug around the wound or on top of intact skin, with the amount absorbed directly related to the duration of application and skin area covered. Lidocaine exerts both its analgesic and local anesthetic effects by blocking sodium channels. Lidocaine within the patch penetrates the skin and stabilizes the neuronal membranes of pain fibers by binding to receptors within sodium channels. These channels have been found to be present in abnormally high numbers in hyperactive or damaged nociceptors. Bound lidocaine blocks the influx of sodium ions, reducing the abnormal ectopic discharges produced by damaged and dysfunctional peripheral nerves ${ }^{20}$, thus interrupting the conduction of the pain signa $^{20}$. However, by diffusion, the concentration of lidocaine reduces by $94 \%$ across the stratum corneum (lidocaine concentration reduced to $0.3 \%$ for a $5 \%$ lidocaine patch $)^{21}$. The lidocaine patch may effectively reduce the pain on the skin just below the patch but theoretically it has almost no analgesic effect on the skin outside the patch. Unless putting the patch over the wound, lidocaine patches may not have effective analgesic effects on the most painful location. Furthermore, the concentration continues to drop by roughly $50 \%$ for each $1 \mathrm{~mm}$ below the stratum corneum ${ }^{21}$. The depth of analgesic effects of the lidocaine patch is not comparable to wound infiltration of lidocaine.

The lidocaine patch formulation is designed to deliver sufficient lidocaine to block sodium channels in nociceptors and reduce the generation of pain impulses, 
but not enough to block sodium channels on large myelinated $A \beta$ fibers $^{22}$. The lidocaine patch therefore produces analgesia at the local site of action without producing local anesthesia, with the skin underneath the patch continuing to have normal sensation. This is known as 'targeted peripheral analgesia' ${ }^{23}$. It is important to contrast this with transdermal patches (e.g. the fentanyl patch) that incorporate a drug delivery system into the patch and depend on absorption of drug into the systemic circulation to produce analgesia. Pharmacokinetic studies have shown that the systemic absorption of lidocaine from the patch is minimal with a mean maximum plasma concentration of $0.13 \mu \mathrm{g} / \mathrm{mL}$, which is about $10 \%$ of the antiarrhythmic dose, resulting in a low risk of systemic side effects ${ }^{23}$. However, the limited systemic absorption means not only minimal systemic side-effects but also minimal analgesic effects systematically.

There are limitations to this meta-analysis. There are a limited number of studies using a lidocaine patch in the acute pain setting and most had a relatively small sample size. The placebo groups in two studies were not completely blinded ${ }^{16,19}$. Also, the etiologies of pain, method of applying lidocaine patches, surgery and study populations were heterogeneous. Adjunct analgesics were not reported in all included studies. Those analgesics may affect the pain scores or opioid consumption.

\section{Conclusion}

This systematic review suggests that the application of a lidocaine patch may not be an effective adjunct for postoperative pain or acute pain management, in terms of postoperative pain scores, opioid consumption and length of hospital stay. Additional large, well designed studies are needed to confirm these findings.

\section{Transparency}

\section{Declaration of funding}

This study was not funded by any company. The authors are paying for priority services.

\section{Declaration of financial or other relationships}

Y.B., T.M., M.T., L.S.-C.L., and T.J.G. have disclosed that they have no significant relationships with or financial interests in any commercial companies related to this study or article.

CMRO peer reviewers on this manuscript have no relevant financial or other relationships to disclose.

\section{Acknowledgments}

The authors thank the faculty and other scientists of their department for their valuable comments.

Previous presentation: This paper was presented in the annual meeting of the Department of Anesthesiology, Duke University, Durham, NC, USA.

\section{References}

1. Kehlet $H$. Effect of postoperative pain treatment on outcome current status and future strategies. Langenbecks Arch Chir 2004;389: 244-9

2. Gan TJ, Habib AS, Miller TE, et al. Incidence, patient satisfaction, and perceptions of post-surgical pain: results from a US national survey. Curr Med Res Opin 2014;30:149-60

3. White PF. Pain management after ambulatory surgery - where is the disconnect? Can J Anesth 2008;55:201-7

4. White PF, Kehlet H. Improving postoperative pain management: what are the unresolved issues? Anesthesiology 2010;112:220-5

5. Shang $A B$, Gan TJ. Optimising postoperative pain management in the ambulatory patient. Drugs 2003;63:855-67

6. Gordon DB, Dahl JL, Miaskowski C, et al. American pain society recommendations for improving the quality of acute and cancer pain management: American Pain Society Quality of Care Task Force. Arch Intern Med 2005; 165:1574-80

7. Vigneault L, Turgeon AF, Cote D, et al. Perioperative intravenous lidocaine infusion for postoperative pain control: a meta-analysis of randomized controlled trials. Can J Anesth 2011;58:22-37

8. Sun $Y$, Li T, Wang N, et al. Perioperative systemic lidocaine for postoperative analgesia and recovery after abdominal surgery: a metaanalysis of randomized controlled trials. Dis Colon Rectum 2012;55: 1183-94

9. Gimbel J, Linn R, Hale M, et al. Lidocaine patch treatment in patients with low back pain: results of an open-label, nonrandomized pilot study. Am J Therapeut 2005;12:311-19

10. Kim KH. Use of lidocaine patch for percutaneous endoscopic lumbar discectomy. Kor J Pain 2011;24:74-80

11. Kim CH, Yoon JU, Lee HJ, et al. Availability of a $5 \%$ lidocaine patch used prophylactically for venipuncture- or injection-related pain in children. J Anesth 2012;26:552-5

12. Gilhooly D, McGarvey B, O'Mahony $H$, et al. Topical lidocaine patch $5 \%$ for acute postoperative pain control. BMJ Case Reports 2011;2011:bcr0620103074

13. Zink KA, Mayberry JC, Peck EG, et al. Lidocaine patches reduce pain in trauma patients with rib fractures. Am Surg 2011;77:438-42

14. Gordon DB, Stevenson KK, Griffie J, et al. Opioid equianalgesic calculations. J Palliat Med 1999:2:209-18

15. Habib AS, Polascik TJ, Weizer AZ, et al. Lidocaine patch for postoperative analgesia after radical retropubic prostatectomy. Anesth Analg 2009;108:1950-3

16. Saber AA, Elgamal MH, Rao AJ, et al. Early experience with lidocaine patch for postoperative pain control after laparoscopic ventral hernia repair. Int J Surg 2009;7:36-8

17. Ingalls NK, Horton ZA, Bettendorf $M$, et al. Randomized, double-blind, placebo-controlled trial using lidocaine patch $5 \%$ in traumatic rib fractures. J Am Coll Surg 2010;210:205-9

18. Kwon YS, Kim JB, Jung HJ, et al. Treatment for postoperative wound pain in gynecologic laparoscopic surgery: topical lidocaine patches. J Laparoendosc Adv Surg Tech Part A 2012;22:668-73

19. Khanna M, Peters C, Singh JR. Treating pain with the lidocaine patch $5 \%$ after total knee arthroplasty. PM\&R 2012;4:642-6

20. Gammaitoni AR, Alvarez NA, Galer BS. Pharmacokinetics and safety of continuously applied lidocaine patches 5\%. Am J Health Syst Pharm 2002;59:2215-20

21. Oshizaka T, Kikuchi K, Kadhum WR, et al. Estimation of skin concentrations of topically applied lidocaine at each depth profile. Int J Pharmaceut 2014;475:292-7

22. Argoff CE. New analgesics for neuropathic pain: the lidocaine patch. Clin $\mathrm{J}$ Pain 2000;16(2 Suppl):S62-6

23. Argoff CE. Targeted topical peripheral analgesics in the management of pain. Curr Pain Headache Rep 2003;7:34-8 